\title{
A NEUROLOGICAL PROBLEM BY AN ANONYMOUS MEMBER.
}

A. B. Male, aged 40, was admitted for recurrent epileptiform attacks of eight years' duration.

Eight and a half years ago, while serving in India as a private in the Royal Fusiliers, he injured his head by falling off a lorry. He sustained concussion and was unconscious for about three hours. He was detained in hospital for I5 days, during which time he felt no untoward symptoms. An X-ray examination of the skull revealed no fracture.

About a month after returning to his regiment he gradually developed various psychoneurotic symptoms. He became depressed and sleepless and took no interest in sport or any other of the activities on which he was previously keen. His efficiency as a soldier became impaired and he got into trouble with the authorities on numerous occasions until it was realised that he was a sick man. He was transferred to a rest hospital for about six weeks and again making a perfect recovery returned to his Unit once more.

Seven months after the head injury, he sustained an epileptic fit while on parade. He felt a premonitory sensation of malaise and fell unconscious. General convulsive movements were present with cyanosis, biting of the tongue and incontinence. On recovery he felt dazed and complained of headache. The next day he was quite well again, but a second fit occurred five days later.

Examination at the Military Hospital revealed no definite abnormality. Whilst under observation two further fits occurred. He was summoned before a Medical Board and discharged from the Army as a case of "Epilepsy".

On his return to England he put himself under his private doctor who treated him with bromides and later, luminal. Fits continued to occur at infrequent intervals, the usual period of freedom being about three months. In all other respects the man felt well and was able to carry out his civilian employment.

Two years ago there was a slight increase in the number of attacks and he was admitted to a Neurological Hospital for investigation. Despite full clinical investigation, together with a further X-ray examination of the skull and of the cerebro-spinal fluid, no evidence of organic disease could be found.

Two months ago he began to notice occasional bouts of twitching of the right side of the face. These occurred for a few minutes at a time, every other day or so. For the same period he had noticed a little tiredness in the right foot; the toe would occasionally catch on the mat or on the kerb. These seemed to get worse.

For a month a little awkwardness had been noticed in the right hand, and writing and shaving were both carried out with some difficulty. To direct questioning, he admitted that on a few occasions he had found some hesitancy in his speech and was at times at a loss for a word.

No headaches were complained of, and the fits had improved in that no attack had occurred for the past three months.

\section{Previous Health.}

The patient had contracted syphilis I4 years ago, and had been thoroughly treated over a period of four years, and was finally pronounced cured.

No other illnesses.

\section{Family History.}

The patient was single.

His brother and parents were alive and healthy. There was no family history of epilepsy. 


\section{Physical Examination.}

This revealed a right-sided hemiparesis most marked in the leg and least noticeable in the arm. The right face was weak, but the tongue did not deviate on protrusion.

Tendon jerks brisker on right than on left side.

Right abdominal responses absent.

Right plantar response extensor.

Sensory tests revealed in the right hand defects of stereognosis, of tactile discrimination and of postural sensibility.

No hemianopia.

Pupils and ocular movements normal. Optic discs healthy.

\section{Diagnosis.}

The following are the chief morbid conditions which present themselves for differentiation in such a case.

Idiopathic Epilepsy. The onset of fits at the age of 32 in a man of a healthy family argues against this diagnosis. The more recent gradual development of a hemiparesis, at a time when the fits were not in evidence suggests a slowly developing intracerebral lesion.

Post-traumatic Epilepsy is suggested by the history of concussion some months prior to the first fit. The recent development of paralysis is difficult to associate with such a diagnosis, unless there is evidence of pressure from a subdural hæmatoma. But an interval of 8 years between the accident and the appearance of paralysis is too long.

Hystero-epilepsy. Despite the history of a psycho-neurosis shortly before the onset of the fits, the essentially epileptic character of the fits, and the hemiparesis with "pyramidal" signs, point clearly to an organic brain affection.

Cerebral Syphilis is a highly probable explanation of the symptoms in this case though actually this diagnosis is excluded (I), by negative serological findings and (2), by an absence of pupillary or other neurological anomalies indicating neurosyphilis.

Cerebral Cysticercosis. This diagnosis is suggested by the late onset of epilepsy and particularly by the man's army service in India. The fact that no history of tapeworm infection could be elicited, and that no subcutaneous nodules could be felt does not exclude this diagnosis. But the lack of calcified cysts in X-ray pictures of the extremities and of the skull, the absence of eosinophilia in the blood and spinal fluid, and the negative specific complement fixation test rule out the possibility of such a condition.

Cerebral Tumour is suggested by the history of fits followed later by paralytic signs and also by the local convulsive movements in the face. The absence of headache, vomiting and papilloedema does not render the diagnosis of tumour inadmissible. This diagnosis is supported by the manometric reading of the spinal fluid pressure, which was greater than $300 \mathrm{~mm}$. Finally, the skiagram of the skull reveals a definite area of calcification in the left parietal lobe of the brain.

All the evidence, therefore, suggests that the cause of the symptoms in this case is a slow growing type of cerebral tumour.

One of three pathological types is most likely in such a case as this: the probabilities lie between an endothelioma (or meningioma), an oligodendroglioma and an astrocytoma. All three are slow-growing and relatively benign types of growth, and in all of which calcification may occur and produce X-ray shadows. 